\title{
Preface and Introduction to the Contents of Issue 49:1
}

\author{
Axel Ruhe
}

Published online: 21 February 2009

(C) Springer Science + Business Media B.V. 2009

This issue starts BIT volume 49. We have a new layout, a variant of the Springer style svjour 3 common to many other journals printed by Springer. Our previous layout was a TeX implementation of our original typesetting, used by Fr. Bagges kgl. Hofbogtrykkeri in Copenhagen from our start in 1961. The TeX style was implemented by Hans Riesel at the Royal Institute of Technology in Stockholm 1986, and I was the first one using the BIT LaTeX style for my paper:

Axel Ruhe, Closest normal matrix finally found! BIT Numer. Math. 27, 585-598 (1987)

I hope this new style will be friendly to our readers and simple to use for our contributors!

Let us see what happens in this issue:

There has been quite a research activity recently to improve the venerable vector norm based condition numbers, expounded by J. H. Wilkinson around 1960, with more precise bounds. In the first paper of this issue, Marc Baboulin and Serge Gratton study componentwise bounds for linear least squares problems. They derive conditions on how much variations in single components in the data perturb the solution. Also the second paper by Coralia Cartis, Nicholas I. M. Gould and Philippe L. Toint is concerned with linear least squares, now the interest is to regularize the solution. Such a regularization needs determination of a parameter, and this is done by solving a secular equation with a special Newton method. The regularization is used in a trust region method for applied nonlinear least squares problems.

\footnotetext{
A. Ruhe $(\varangle)$

Department of Numerical Analysis and Computer Science (NADA), Royal Institute of Technology (KTM), 10044 Stockholm, Sweden

e-mail: ruhe@kth.se
} 
Stefano De Marchi, Marco Vianello, and Yuan Xu deal with cubature and the related hyperinterpolation problem in higher dimensions. A Clenshaw Curtis type algorithm is computed by means of a 3 dimensional fast Fourier transform.

Harri Hakula and Nuutti Hyvönen describe a method for electrical impedance tomography a practically important inverse problem. They use test dipoles to model interior inhomogeneities, measured on the surface of an object. The connection between this complete electrode model, CEM, and the idealized continuum model, CM, is investigated.

The rest of papers deal with time stepping for dynamic systems. Laura Hewitt and Adrian Hill study the algebraic stability of general linear methods, GLM, to solve a system of ordinary differential equations, ODE. The theory of discrete algebraic Riccati equations is used to derive a stability condition formulated as a matrix equation. Some algebraically stable methods of higher accuracy are derived.

Arnulf Jentzen and Peter E. Kloeden develop methods for random ordinary differential equations, RODE. Even in this case, when the driving vector field is only Hölder continuous, it is possible to develop higher order schemes, using an integral Taylor expansion.

Georg Kitzhofer, Othmar Koch and Ewa Weinmüller develop an algorithm for a parameter dependent singular two point boundary value problem, BVP. Path following for different values of the parameter is done by semi arc length continuation and the BVPs are solved by polynomial collocation on adaptive grids. The technique is illustrated on blow up solution profiles of the Ginzburg Landau equation.

René Lamour and Francesca Mazzia develop an algorithm to compute consistent initial values for a differential algebraic system, DAE, of index up to 3 . It is crucial how the derivative is approximated.

Robert McLachlan, Reinout Quispel, and Priscilla Tse study numerical integrators for ODE systems that preserve linear behavior close to a stationary point. Two self adjoint linearity preserving B-series methods are developed and tested on Hamiltonian ODE systems.

Christof Neuhauser and Mechthild Thalhammer use high order exponential operator splitting methods to integrate the time dependent Schrödinger equation with a possibly unbounded potential.

Thorsten Sickenberger, Ewa Weinmüller and Renate Winkler develop a local error estimator, based on defect correction, that can be used for data that are only moderately smooth. It is usable for stochastic differential equations and differential algebraic equations with small noise.

Welcome to the BIT of 2009 !

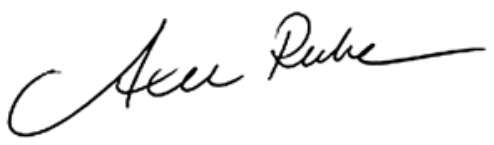

Axel Ruhe 